\title{
CEP215 and AURKA regulate spindle pole focusing and aMTOC organization in mouse oocytes
}

\author{
Xiaotian Wang, Claudia Baumann, Rabindranath De La Fuente and Maria M Viveiros \\ Department of Physiology and Pharmacology, College of Veterinary Medicine, University of Georgia, Athens, \\ Georgia, USA \\ Correspondence should be addressed to M M Viveiros; Email: viveiros@uga.edu
}

\begin{abstract}
Acentriolar microtubule-organizing centers (aMTOCs) play a critical role in stable meiotic spindle assembly in oocytes, necessary for accurate chromosome segregation. Yet, there is a limited understanding of the essential regulatory components of these unique MTOCs. In somatic cells, CEP215 (Centrosomal Protein 215) serves as an important regulator of centrosome maturation and spindle organization. Here, we assessed whether it has a similar function in mouse oocytes. CEP215 was detected in oocyte lysates and specifically localized to aMTOCs throughout the progression of meiosis in a pericentrin-dependent manner. Super-resolution microscopy revealed CEP215 co-localization with pericentrin and a unique pore/ring-like structural organization of aMTOCs. Interestingly, inhibition of Aurora Kinase A in either MI or MII-stage oocytes resulted in a striking loss of the ring-like aMTOC organization and pronounced CEP215 clustering at spindle poles, as well as shorter spindles with highly focused poles. In vitro siRNA-mediated transcript knockdown effectively reduced CEP215 in approximately $85 \%$ of the oocytes. Maturation rates to MII were similar in the Cep215 siRNA and injected controls; however, a high percentage ( 40\%) of the Cep215-knockdown oocytes showed notable variations in spindle pole focusing. Surprisingly, pericentrin and $\gamma$-tubulin localization and fluorescence intensity at aMTOCs were unaltered in knockdown oocytes, contrasting with mitotic cells where CEP215 depletion reduced $\gamma$-tubulin at centrosomes. Our results demonstrate that CEP215 is a functional component of oocyte aMTOCs and participates in the regulation of meiotic spindle pole focusing. Moreover, these studies reveal a vital role for Aurora Kinase A activity in the maintenance of aMTOC organization in oocytes.

Reproduction (2020) 159 261-274
\end{abstract}

\section{Introduction}

Chromosome segregation errors that occur during meiotic division can result in aneuploid oocytes and developing embryos upon fertilization (Hassold \& Hunt 2001, Nagaoka et al. 2012). The accuracy of chromosome segregation relies on the formation of a stable spindle apparatus and the establishment of correct chromosome-microtubule interactions (Chmátal et al. 2015). Interestingly, meiotic spindle assembly and organization in mammalian oocytes differ from mitosis (Schuh \& Ellenberg 2007, Baumann et al. 2017); yet, the underlying mechanisms that regulate this essential process are not well defined. In somatic cells, centrosomes function as the primary organizing centers for the formation and anchorage of spindle microtubules (Doxsey et al. 2005) and are composed of two centrioles surrounded by a protein matrix of pericentriolar material (PCM), required for microtubule nucleation. However, mammalian (human and mouse) oocytes lose centrioles early in development (Szollosi et al. 1972, Sathananthan et al. 2000, Manandhar et al. 2005) and, thus, lack canonical centrosomes during meiotic division. Nevertheless, studies show that mouse oocytes express essential PCM proteins, despite the absence of centrioles (Maro et al. 1985, Combelles \& Albertini 2001, Manandhar et al. 2005). These unique acentrioloar MTOCs (aMTOCs) nucleate microtubules and contribute to meiotic spindle assembly (Schuh \& Ellenberg 2007, Ma \& Viveiros 2014, Baumann et al. 2017). Key oocyte aMTOC-associated proteins include $\gamma$-tubulin, which catalyzes the assembly of spindle microtubules, as well as pericentrin (Combelles \& Albertini 2001, Schuh \& Ellenberg 2007).

Pericentrin (PCNT) functions as a core scaffolding protein that binds key PCM proteins, necessary for centrosome maturation and spindle assembly in somatic cells (Zimmerman et al. 2004, Delaval \& Doxsey 2010). To assess PCNT and aMTOC function in oocytes, we previously developed an oocyte-conditional Pcntknockdown mouse model and demonstrated that meiotic division is highly error-prone in the absence of PCNT (Baumann et al. 2017). Pericentrin-depleted oocytes show disruptions in meiotic spindle assembly and organization with increased chromosome errors and aneuploidy leading to poor female fertility. 
Notably, depletion of PCNT results in the loss of key aMTOC-associated proteins from meiotic spindle poles, including $\gamma$-tubulin and the $\gamma$ TuRC adaptor protein NEDD1 (Baumann et al. 2017). Thus, establishing that PCNT functions as a crucial scaffolding protein in oocyte aMTOCs, analogous to its role in canonical centrosomes (Zimmerman et al. 2004, Delaval \& Doxsey 2010). Considering the major role of aMTOCs in stable meiotic spindle assembly in oocytes, it is essential to gain a better understanding of the key associated proteins and to determine how potential disruptions contribute to meiotic errors.

Analysis of our oocyte-conditional Pcnt-knockdown mouse model revealed that PCNT regulates the localization of cyclin-dependent kinase-5 regulatory subunit-associated protein 2 (CDK5RAP2) at metaphase II (Baumann et al. 2017). CDK5RAP2, also known as CEP215 (Centrosomal Protein 215), is the vertebrate homologue of Drosophila Centrosomin (Cnn) (Kim \& Rhee 2014) and is conserved from yeast to humans (Kraemer et al. 2011). The protein is widely expressed in somatic cells, with high levels in the brain and recessive gene mutations linked to primary microcephaly in humans (Bond et al. 2005). Similarly, Cdk5Rrap2 $2^{\text {an/an }}$ mutant mice show reduced cerebral cortex size due to altered mitotic orientation and abnormal numbers of spindle poles in neuronal progenitor cells (Lizarraga et al. 2010). Mitotic errors have also been linked to the loss of germ cells during development in these mutants (Russell et al. 1985, Zaqout et al. 2017). CEP215 localizes to the centrosome at all stages of the mitotic cell cycle and interacts with $\gamma$-tubulin and PCNT, playing a role in microtubule nucleation as well as centrosome maturation (Fong et al. 2008, Buchman et al. 2010, Choi et al. 2010, Kraemer et al. 2011). Moreover, CEP215 together with ninein and centriolin have been identified as a subset of key proteins that are targeted to mitotic spindle poles by PCNT, and they support microtubule anchoring as well as the formation of mature spindle poles in somatic cells (Chen et al. 2014). Whether CEP215 function is conserved in pre-ovulatory oocyte aMTOCs is not known. Hence, in this study we evaluated CEP215 expression during the progression of meiosis and tested its function in mouse oocytes.

\section{Materials and methods}
Animals
All mice were housed at a constant temperature $\left(24-26^{\circ} \mathrm{C}\right)$ and under a controlled light cycle (12 h light:12 h darkness) with food and water provided ad libitum. All animal use protocols were approved by the 'Institutional Animal Care and Use Committee' (IACUC) at the University of Georgia (Athens, GA, USA), and the experiments were conducted in accordance with all specified guidelines.

\section{Oocyte collection and culture}

The subcellular distribution of CEP215 was assessed during meiotic division in oocytes obtained from B6D2F1 mice (C57BL/6 J females $\times \mathrm{DBA} / 2 \mathrm{~J}$ males). For some experiments, oocytes were also collected from transgenic (Tg) mice with an oocyte-conditional knockdown of pericentrin (Baumann et al. 2017). To promote pre-ovulatory follicle development, 20- to 23-day-old females were injected with $5 \mathrm{IU}$ pregnant mare serum gonadotrophin (EMD Biosciences, La Jolla, CA, USA) and the ovaries were collected 44-48 h later. Cumulus celloocyte complexes were released from follicles in minimal essential medium (MEM) supplemented with $3 \mathrm{mg} / \mathrm{mL}$ BSA (Sigma Aldrich) and $1 \mu \mathrm{g} / \mathrm{mL}$ milrinone (Sigma Aldrich), a phosphodiesterase inhibitor to maintain the oocytes in prophase-I arrest (GV-stage). After a short (1.5 h) incubation, the surrounding cumulus cells were removed by gentle pipetting and the denuded GV-stage oocytes were transferred to fresh milrinone-free media. Oocytes were cultured at $37^{\circ} \mathrm{C}$ in MEM/BSA with 5\% fetal calf serum (Hyclone, Logan, UT) for specific times. Oocytes arrested at prophase-I, germinal vesicle breakdown (GVB), as well as those at prometaphase, Metaphase-I (MI) and Metaphase-II (MII) stages were fixed following a 0, 2, 4, 8, and 17 h culture, respectively. For some experiments, in vivo matured MII eggs were collected from the oviducts of superovulated female mice approximately $16 \mathrm{~h}$ after the administration of $5 \mathrm{IU}$ human Chorionic Gonadotrophin (EMD Biosciences).

\section{Immunofluorescence analysis}

Oocytes were fixed and immunolabeled with specific antibodies as previously described (Baumann \& Viveiros 2015). In brief, the oocytes were fixed with $4 \%$ paraformaldehyde in Dulbecco's phosphate buffered saline (DPBS) with $0.05 \%$ Triton-X for $35 \mathrm{~min}$ at $37^{\circ} \mathrm{C}$, then washed and blocked in DPBS supplemented with $5 \%$ serum. The wash buffer was supplemented with a $1 \%$ antibiotic and antimycotic solution and $0.02 \%$ Triton-X. Specific primary antibodies were used to detect CEP215 (Millipore) (1/250 dilution), Pericentrin (BD Biosciences, San Jose, CA and Covance, Princeton, NJ, USA) (1/1000 dilution), $\gamma$-tubulin (Sigma Aldrich, 1/500 dilution), and acetylated $\alpha$-tubulin (Sigma Aldrich, 1/1000 dilution). Alexa Fluor-conjugated 488 and 555 secondary antibodies were purchased from Life Technologies (Fisher, Eugene, OR, USA). DNA was counterstained in Vectashield mounting media containing DAPI (Vector Laboratories, Burlingame, CA, USA). Fluorescence intensity was assessed using an upright fluorescent microscope and imaging software (Leica Microsystems). In addition, high resolution structured illumination microscopy (SR-SIM) analysis was undertaken using a Zeiss ELYRA S1 system at the Biomedical Microscopy Core (BMC) facility, University of Georgia.

\section{Western blotting}

After removal of the surrounding granulosa cells, denuded GV and MII oocytes were frozen in RIPA buffer $(1 \mathrm{M}$ Tris- $\mathrm{HCl}$ $\mathrm{pH} 7.4,5 \mathrm{M} \mathrm{NaCl}$, $5 \%$ sodium deoxycholate, $10 \%$ SDS, $10 \%$ 
Nonidet P40, and 0.5 M EGTA) supplemented with a protease and phosphatase inhibitor solution (Thermo Scientific). Prior to analysis the samples were thawed on ice, mixed with $5 \times$ loading buffer, and heated at $98^{\circ} \mathrm{C}$ for $7 \mathrm{~min}$. The proteins were separated in $8 \%$ acrylamide gels containing 10\% SDS, then transferred onto hydrophobic PVDF membranes (Millipore). Following transfer, the membrane was blocked in TBS supplemented with $2 \%$ Tween 20 (TBST) and 5\% milk powder, then incubated with anti-CEP215 (Millipore, $1 / 1000$ dilution) at $4^{\circ} \mathrm{C}$ overnight, and subsequently washed in TBST. A peroxide-conjugated secondary antibody (Jackson ImmunoResearch) was added for $1 \mathrm{~h}$ and the membrane was then washed in TBST. An ECL kit (Millipore) was used for chemiluminescent detection. The membrane was also probed with anti- $\beta$ tubulin (Sigma Aldrich, 1/2000 dilution) as a control, under similar conditions. Individual band intensity was quantified, for three independent experimental replicates, using the Image J software, and the relative total protein values in each group were compared to the control, which was normalized to 1.0 .

\section{Incubation with nocodazole or Aurora Kinase A (AURKA) inhibitor}

To test whether CEP215 localization to aMTOC upon the resumption of meiosis is dependent on microtubules, GV-stage oocytes were cultured for $2 \mathrm{~h}$ in MEM/BSA with 1 $\mu \mathrm{M}$ Nocodazole (Sigma Aldrich) to destabilize microtubules, while control oocytes were cultured in media alone. One group of oocytes was fixed immediately following the $2 \mathrm{~h}$ in vitro maturation, while another group was carefully washed $3 \times$ to remove nocodazole and transferred to fresh media. These oocytes were placed in culture and subsequently fixed either at $15 \mathrm{~min}$ and $1 \mathrm{~h}$ post washing. Immunofluorescence was used to assess microtubule formation and CEP215 distribution, including the number of CEP215 foci. In addition, we tested whether CEP215 localization at aMTOCs, in either MI or MII-stage oocytes, is regulated by Aurora Kinase A (AURKA). Ovulated MII oocytes were collected as previously described and cultured for $4 \mathrm{~h}$ in MEM/BSA supplemented with 500 nM MLN8237 (Selleckchem, Houston, TX, USA), a selective inhibitor for AURKA (Sloane et al. 2010) previously used in mouse oocytes at this concentration (Bury et al. 2017). To test AURKA inhibition at the Ml-stage, GV-stage oocytes were collected and cultured for $7 \mathrm{~h}$ in MEM/BSA for maturation to $\mathrm{MI}$, then subsequently incubated in media supplemented with $500 \mathrm{nM}$ MLN8237. Control MI and MII oocytes were incubated in media alone under similar conditions. All cultures were maintained at $37^{\circ} \mathrm{C}$ as previously described, and the oocytes were subsequently fixed for immunofluorescence analysis to assess CEP215 distribution and fluorescence intensity, as well as chromosome and microtubule configurations.

\section{Cep215 transcript knockdown using specific siRNAs}

To test CEP215 function, a mix of four specific pre-designed and validated siRNAs directed against the mouse Cep215 gene sequence (Qiagen) were microinjected into GV-arrested (prophase-I) oocytes to knockdown Cep215 transcripts.
Two control groups were included: (1.) non-injected oocytes that were subject to the same culture conditions and (2.) oocytes injected with control non-specific siRNAs (Qiagen). The siRNAs were microinjected into the cytoplasm of denuded GV oocytes in M2 medium with $1 \mu \mathrm{g} / \mathrm{mL}$ milrinone, as previously described (Ma et al. 2010). Injection volumes were standardized using Femtotip capillaries and a Femptojet micro-injector (Eppendorf, Hauppauge, NY, USA). The oocytes were maintained in prophase-I arrest for $30 \mathrm{~h}$ in milrinone supplemented MEM/BSA with 5\% serum (Hyclone) to enable sufficient transcript knockdown. The oocytes were then washed and transferred into milrinone-free media for an additional $17 \mathrm{~h}$ culture to undergo meiotic maturation. Each group of oocytes was fixed to assess CEP215 fluorescence intensity, chromosome and meiotic spindle configurations, as well as key aMTOC-associated proteins (pericentrin and $\gamma$-tubulin) in individual control and Cep215-depleted oocytes.

\section{Statistical analysis}

All data are presented as mean values ( \pm S.E.M.) from at least three independent experimental replicates. The GraphPad Prism software was used for data analysis and preparation of graphs. The data were analyzed by either ANOVA or $t$-test for comparison among groups, and differences were considered to be significant when $P<0.05$.

\section{Results \\ CEP215 localizes specifically to oocyte aMTOCs in a pericentrin (PCNT)-dependent manner}

The subcellular distribution of CEP215 in control oocytes was assessed by immunofluorescence during the progression of meiosis, while total protein levels were determined by Western blot at GV and MII stages. Pre-ovulatory oocytes arrested at prophase-I (GV-stage) showed a bright CEP215 signal at a single aMTOC (Fig. 1A, a arrowhead), whereas during metaphase-II CEP215 was detected at the spindle poles (Fig. 1A, b arrowheads) as well as at distinct cytoplasmic aMTOCs (Fig. 1A and D). Western blot analysis of oocyte lysates revealed a double band of $\sim 250 \mathrm{kDa}$ corresponding to CEP215 in both GV and MII-stage oocytes (Fig. 1B). Total protein levels tended to increase at MII, suggesting an upregulation during meiotic maturation, although the difference was not significant (Fig. 1C). CEP215 distribution was also assessed at key stages of meiotic maturation. As oocytes resumed meiosis and assembled the first meiotic spindle at early MI (Fig. 1D, a-d), CEP215 clustered at the forming spindle poles. During both $\mathrm{MI}$ and MII, CEP215 localized specifically to the aMTOCs in a ring-like structure at the meiotic spindle poles (Fig. 1D, e-l) with no labeling detected along the spindle microtubules.

Analysis by super resolution microscopy (SR-SIM) revealed CEP215 co-localization with PCNT at aMTOCs (Fig. 2A). The proteins co-localize in a seemingly 

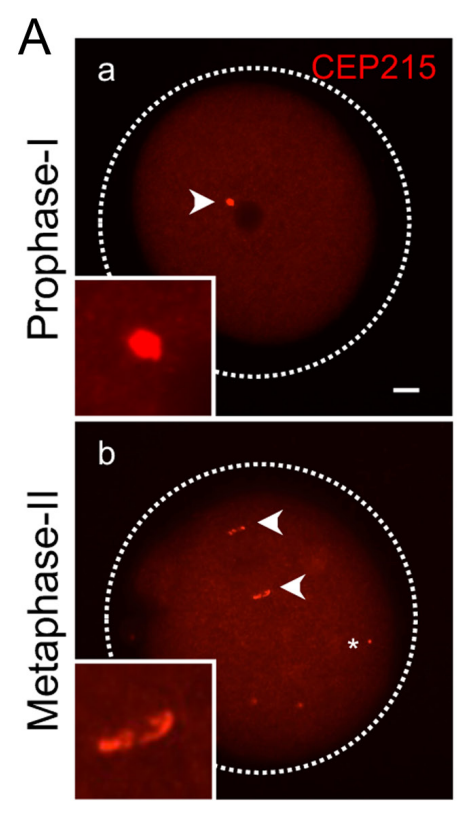

D
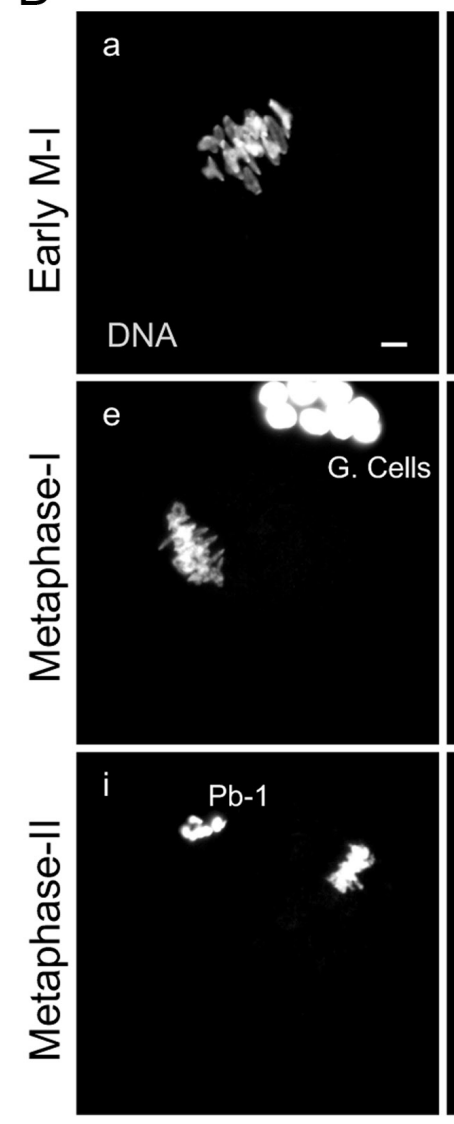

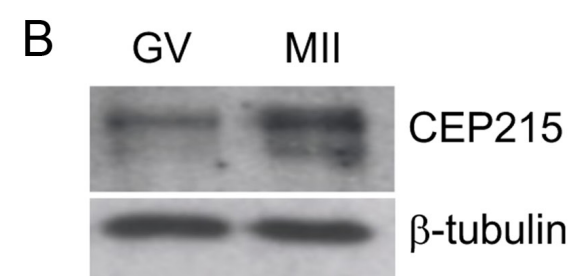

C

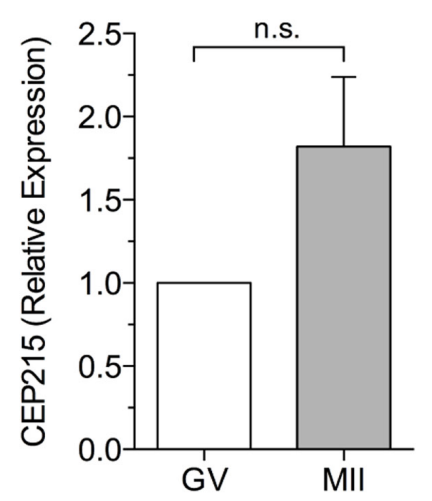

Figure 1 CEP215 expression and localization at oocyte aMTOCs during meiotic division. (A) Mouse oocytes were collected for immunofluorescence to assess the subcellular distribution of CEP215 (red) during prophase-I arrest (GV, $n=57)$ and metaphase-II (MII, $n=65)$. (B) Western blot of CEP215 and $\beta$-tubulin total protein levels in lysates from GV and MII-stage oocytes (50 oocytes/lane). (C) Total CEP215 in MII oocytes relative to the GV-stage, which was normalized to 1.0 ( $n=3$ replicates). (D) Oocytes at early MI $(n=84)$, MI $(n=74)$, and MII $(n=65)$ were double-labeled with specific anti-CEP215 (red, arrows) and anti-acetylated $\alpha$-tubulin (green) antibodies to detect microtubules. CEP215 (red) localizes specifically at the spindle poles (arrows). DAPI-labeled DNA is shown in grey. Pb-1: Polar body. G. Cells: Granulosa cells. *Cytoplasmic aMTOCs. Insets show $2 \times$ and $4 \times$ (prophase-I) magnifications of the spindle pole area. Scale bar of $10 \mu \mathrm{m}$. 

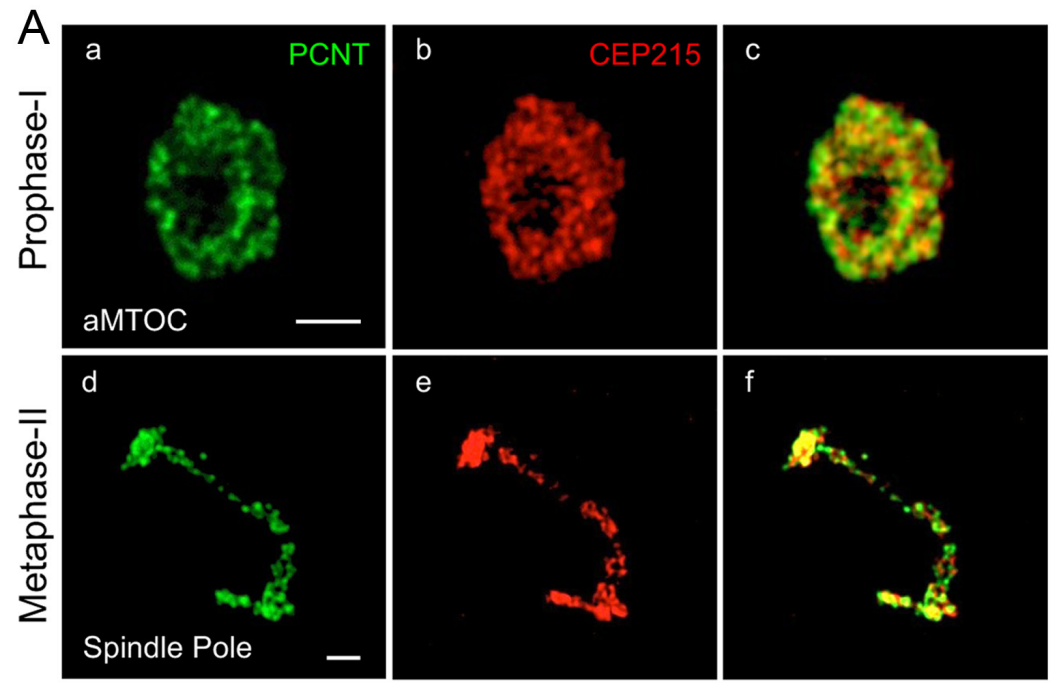

\section{B}

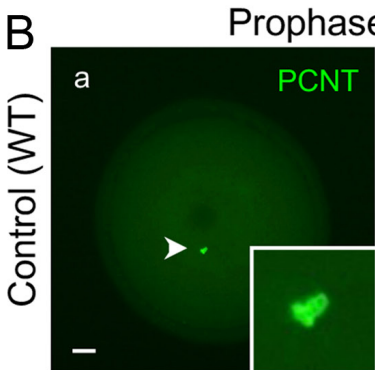

-I (GV)
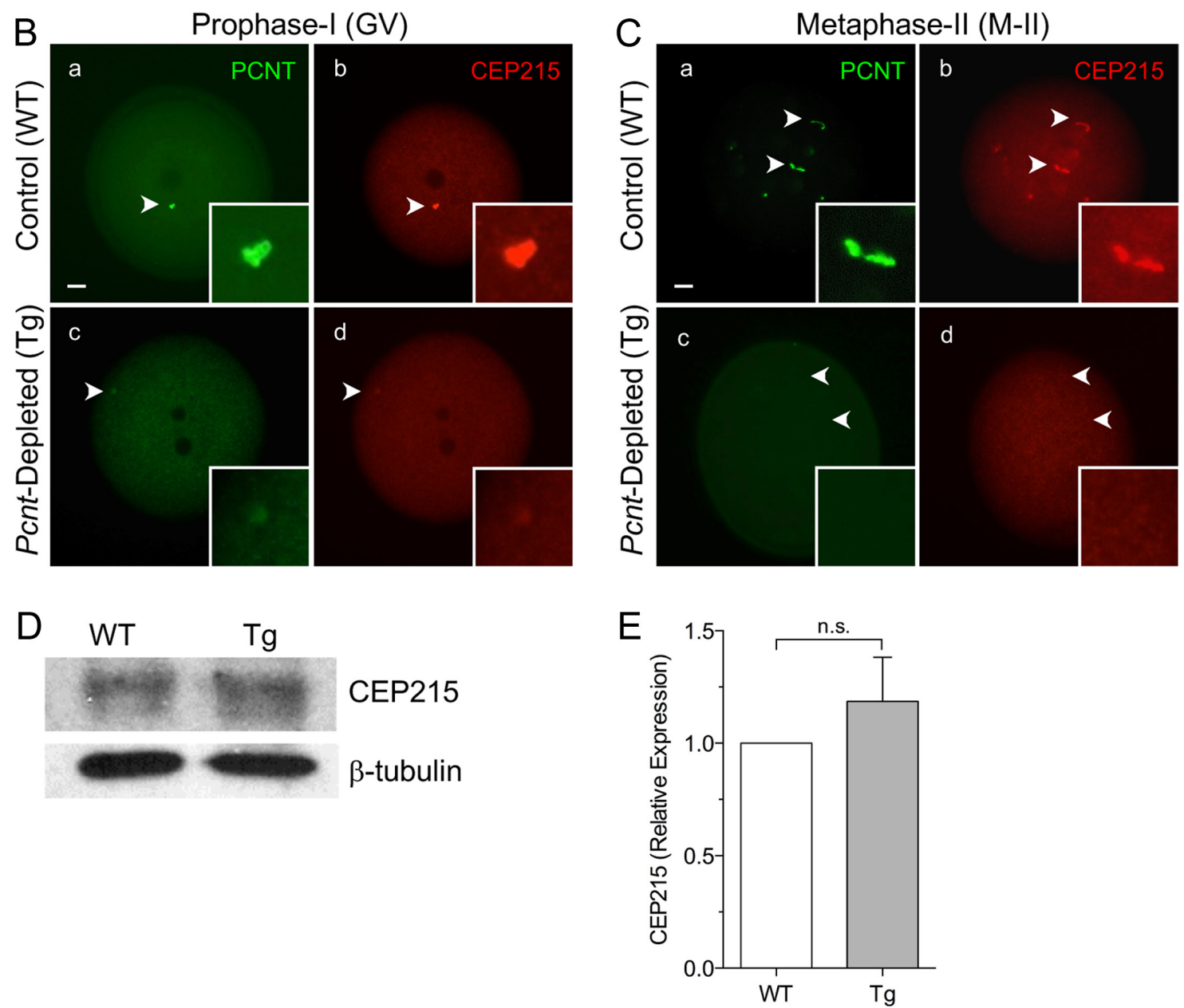

Figure 2 CEP215 localization to oocyte aMTOCs is dependent on PCNT. (A) High resolution (SR-SIM) microscopy analysis of CEP215 (red) and PCNT (green) co-localization at aMTOCs in prophase-I arrested $(n=6)$ and ovulated MII $(n=6)$ oocytes. Scale bars of $1 \mu \mathrm{m}$. (B) Prophase-I $(n=125)$ and (C) MII-stage $(n=107)$ control and Pcnt-depleted oocytes collected from WT and Tg mice, respectively, were double-labeled with specific anti-CEP215 (red) and anti-PCNT (green) antibodies. Insets show $2 \times$ and $4 \times$ (prophase-l) magnifications of aMTOCs. Scale bar of $10 \mu \mathrm{m}$. (D) Western blot of CEP215 and $\beta$-tubulin protein levels in control and Pcnt-depleted MII oocyte lysates (50 oocytes/lane) collected from WT and Tg mice, respectively. (E) Total CEP215 in Pcnt-depleted oocytes from Tg mice compared WT controls, which was normalized to 1.0 ( $n=3$ replicates). 
interlacing pattern, both at prophase-I and at the meiotic spindle poles in MII-stage oocytes. Interestingly, in prophase-I oocytes the aMTOC-associated proteins form a small 'pore-like' structure, suggestive of possible higher order organization. At MII, these proteins were also observed in a partial, or complete, large ring structure. To determine if CEP215 localization to aMTOCs is dependent on PCNT, we assessed CEP215 expression in control and PCNT-depleted oocytes from transgenic mice with oocyte-conditional knockdown of Pcnt. We previously reported the absence of CEP215 in PCNT-depleted MII oocytes (Baumann et al. 2017). Here, we extended these findings by assessing CEP215 distribution in prophase-I arrested oocytes and evaluating total protein levels in control (WT) and Tg oocytes (Fig. 2B, C, D and E). Notably, CEP215 was not detected at aMTOCs in PCNT-depleted oocytes at either prophase-I or MII-stages (Fig. 2B and C). Yet, Western blotting demonstrated similar total CEP215 levels in control (WT) and PCNT-depleted MII oocytes from Tg mice (Fig. 2D and E). Thus, loss of PCNT specifically disrupts the localization of CEP215 to aMTOC, but not the total protein levels. These data support that CEP215 is a conserved protein component of aMTOCs in mammalian oocytes during meiotic maturation and that its localization to aMTOCs is PCNT dependent.

\section{Aurora Kinase $A$ activity and microtubules affect CEP215 distribution at aMTOCs}

Studies show that the formation and organization of multiple aMTOCs upon the resumption of meiosis in oocytes is dependent on microtubules (Schuh \& Ellenberg 2007, Clift \& Schuh 2015). To test whether CEP215 accumulation at aMTOCs is similarly regulated, we inhibited microtubule polymerization with nocodazole (a microtubule depolymerizing agent) in oocytes undergoing the resumption of meiosis (GVB). After a 2 h culture, all control oocytes exhibited microtubule formation around the condensing chromosomes (Fig. $3 \mathrm{~A}$ and $\mathrm{B}$ ), together with multiple aMTOCs labeled with CEP215 (Fig. 3A and C). In contrast, the majority of nocodazole-treated oocytes lacked microtubules after $2 \mathrm{~h}$ (Fig. 3A, e-h and B) and few $(<20 \%)$ contained multiple aMTOCs denoted by CEP215 foci (Fig. 3C). Instead, CEP215 was primarily detected at one or two foci only (Fig. 3A, g and C) in the absence of microtubules. After washing off nocodazole and transferring to fresh media, MTs were observed in all oocytes within $15 \mathrm{~min}$. By $1 \mathrm{~h}$ post wash, a pronounced MT array was noted around the chromosomes, although spindle formation was seemingly delayed compared to the control oocytes. Concurrently, multiple CEP215 foci were detected in all oocytes within $15 \mathrm{~min}$ of nocodazole removal (Fig. 3A and C). At this time, the total (mean \pm S.E.M.) number of CEP215 foci was higher in the nocodazole wash-off group, compared to the control oocytes that showed CEP215 accumulation in larger areas. With the re-organization of CEP215, there were fewer total foci detected in both groups after the $1 \mathrm{~h}$ culture (Fig. 3C). This observation is consistent with the organization of PCNT foci in mouse oocytes following the resumption of meiosis and microtubule formation (Schuh \& Ellenberg 2007).

Next, we tested the regulation of CEP215 localization at aMTOCs in fully assembled meiotic spindles of ovulated MII oocytes. Phosphorylated (active) Aurora Kinase A (pT288 AURKA) localizes to aMTOCs in oocytes (Ding et al. 2011, Solc et al. 2012, Bury et al. 2017) as well as centrosomes in somatic cells, where it plays a role in centrosome maturation (Sardon et al. 2008). Moreover, in Drosophila, the ortholog of CEP215 (Cnn) interacts with AURKA (Terada et al. 2003). Therefore, we assessed CEP215 distribution and spindle structure in ovulated MII oocytes following a $4 \mathrm{~h}$ culture in media alone (controls) or with an Aurora A pharmacological inhibitor (MLN8237). In control oocytes, CEP215 was detected at the assembled spindle pole aMTOCs in a partial or full ring configuration, and all chromosomes were congressed at the metaphase plate (Fig. 4A). In contrast, oocytes incubated with MLN8237 showed significant alterations in CEP215 distribution, spindle structure, and chromosome alignment. Bright CEP215 labeling, with no difference in overall fluorescence intensity (Fig. 4D), was still detected at the spindle poles, but in a tightly focused and significantly smaller area (Fig. 4C). Pericentrin was also observed in a similar tightly compact area (Fig. 4A, $\mathrm{i}-\mathrm{I}$ ), indicating that the inhibition of Aurora A disrupts the overall organization of aMTOCs at assembled spindle poles. Super-resolution (SR-SIM) microscopy (Fig. 4B) revealed a complete loss of the ring-like aMTOC structure in response to AURKA inhibition, such that CEP215 was detected as small foci with no discernable organization. MLN8237-treated oocytes also exhibited high rates ( 50\%) of chromosome misalignment (Fig. 4E), with one or more chromosomes not congressed at the metaphase plate, suggestive of potential chromosome attachment errors. Moreover, the treated oocytes contained shorter spindles with highly focused (narrow) spindle poles. Quantitative analysis confirmed a significant decrease in the mean spindle length (Fig. 4F) as well as a pronounced decrease in the average spindle pole width (Fig. 4G), such that the ratio of pole width to spindle length (Fig. $4 \mathrm{H}$ ) was also significantly lower. MI oocytes treated with MLN8237 showed similar alterations, exhibiting significantly shorter spindles with highly focused poles, as well as loss of aMTOC organization with bright CEP215 and increased chromosome misalignment (Supplementary Fig. 1, see section on supplementary materials given at the end of this article). These data demonstrate that inhibition of Aurora A during either MI or MII significantly disrupts assembled meiotic spindle aMTOC organization, CEP215 distribution, and meiotic spindle size. 
A
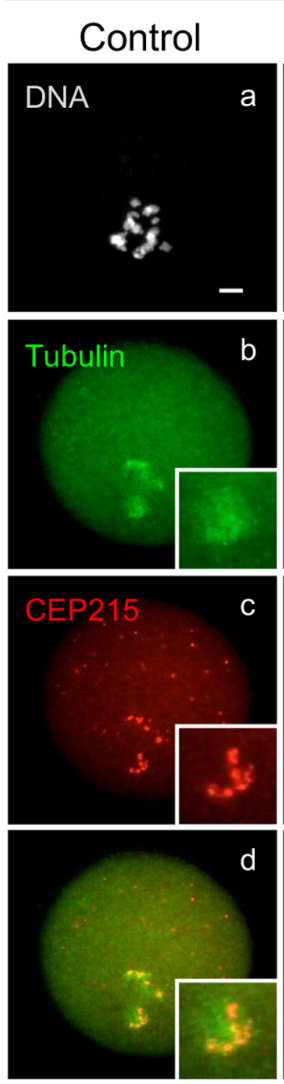

d

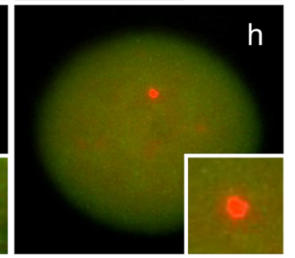

B

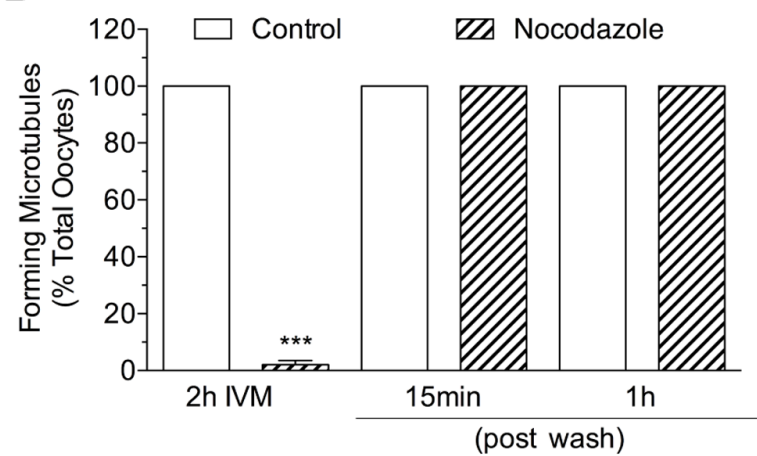

15min (post wash)

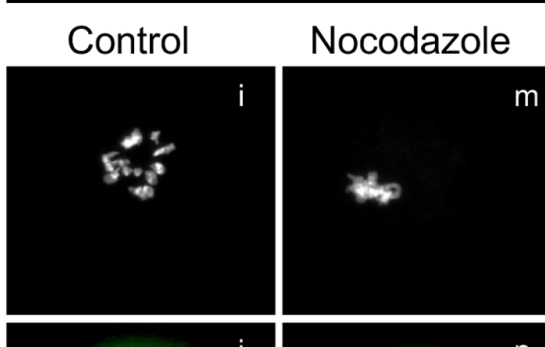

f

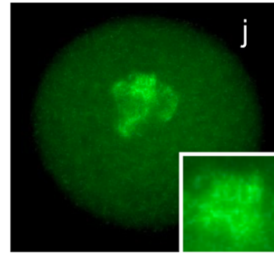

g
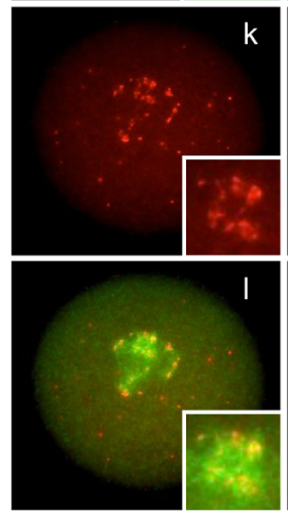

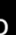

$\frac{1 \mathrm{~h} \text { (post wash) }}{\text { Control Nocodazole }}$
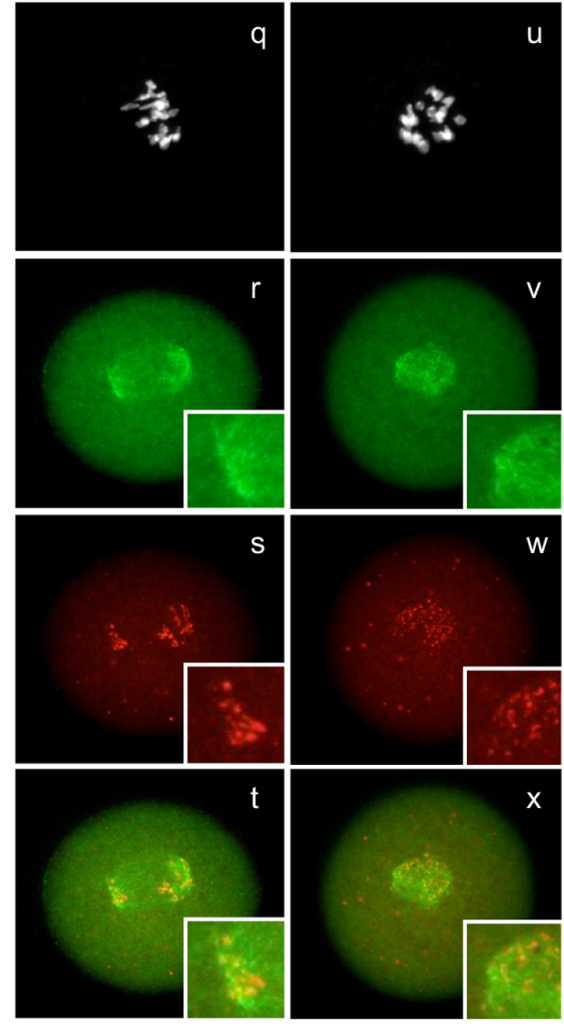

C

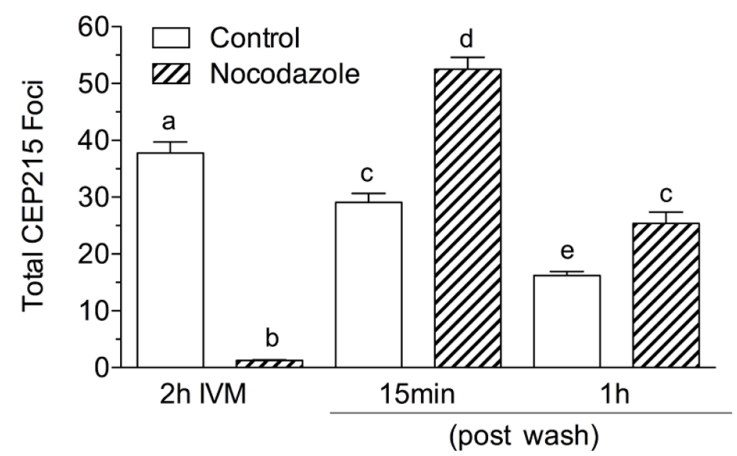

Figure 3 CEP215 localization to oocyte aMTOCs relies on intact microtubules upon meiotic resumption. (A) Representative images of control ( $n=45$ per group) and nocodazole-treated $(1 \mu \mathrm{M})$ oocytes $(n=55$ per group) following a $2 \mathrm{~h}$ culture, as well as 15 min and $1 \mathrm{~h}$ post washing and transfering to fresh media alone. Oocytes were double-labeled with specific anti-CEP215 (red, arrows) and anti-acetylated $\alpha$-tubulin (green) antibodies. The percent (mean \pm S.E.M.) total oocytes containing (B) forming spindle microtubules and (C) total number (mean \pm S.E.M.) of CEP215 foci corresponding to multiple aMTOCs, between control and Nocodazole-treated oocytes ( $n=3$ replicates). DAPI labeled DNA is shown in grey. Insets show $2.5 \times$ magnifications. Scale bar of $10 \mu \mathrm{m}$. ${ }^{* * *} P<0.001$.

\section{Knockdown of Cep215 transcripts disrupts meiotic spindle pole focusing}

To test function, Cep215 transcripts were knocked down in oocytes using specific siRNAs directed against the mouse coding sequence. Analysis of individual oocytes in all groups revealed a significant decrease in CEP215 fluorescence intensity (Fig. 5A) in the Cep215
siRNA group relative to the controls, indicating efficient knockdown. The majority of oocytes $(\sim 85 \%)$ injected with Cep215 siRNAs showed little to no CEP215 labeling at the meiotic spindle poles (Fig. 5A and B). At the end of a $17 \mathrm{~h}$ culture, DAPI-labeled DNA configurations were assessed to determine the progression of meiosis (Fig. 5C). Over $98 \%$ of oocytes in all groups underwent GVB (data not shown), indicating no significant effect 

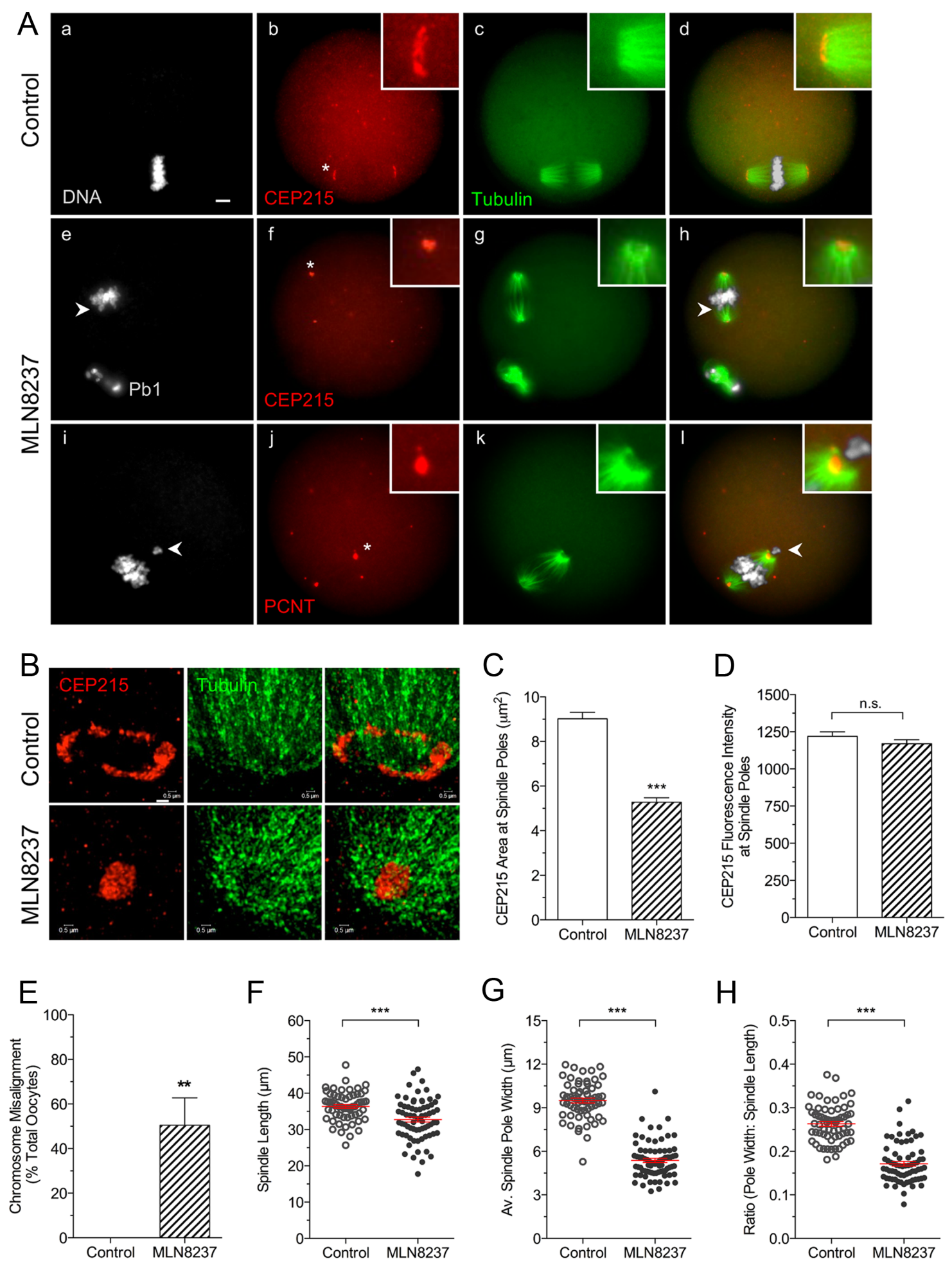

Figure 4 Aurora Kinase A regulates aMTOC organization and CEP215 clustering at assembled meiotic spindle poles. (A) Representative images of ovulated MII control $(n=62)$ and MLN8237-treated (500 nM) oocytes $(n=70)$ following a $4 \mathrm{~h}$ culture. The oocytes were double-labeled with specific anti-CEP215 (a-h, red, arrows) or anti-PCNT (i-l) together with anti-acetylated $\alpha$-tubulin (green) antibodies. (B) High resolution (SR-SIM) microscopy of CEP215 (red) and microtubules (green) at spindle pole aMTOCs in control and MLN8237-treated oocytes. Mean ( \pm S.E.M.) comparisons of the (C) CEP215 area at the spindle poles as well as (D) CEP215 fluorescence intensity, and (E) the percent total oocytes with chromosome misalignment errors, between the control and MLN8237-treated oocytes. Quantitative analysis (mean \pm S.E.M.) of the (F) meiotic spindle length, $(\mathrm{G})$ average pole width, and $(\mathrm{H})$ ratio of the pole width to spindle length measured in the control and MLN8237-treated oocytes ( $n=3$ replicates). DAPI labeled DNA is shown in grey. Insets show $2 \times$ magnifications. Scale bar of $10 \mu$ m. ${ }^{* *} P<0.01$ and ${ }^{* * *} P<0.001$. 


\section{A}

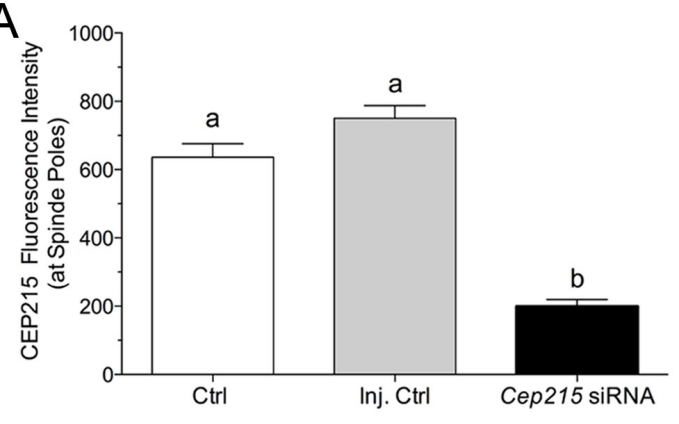

B
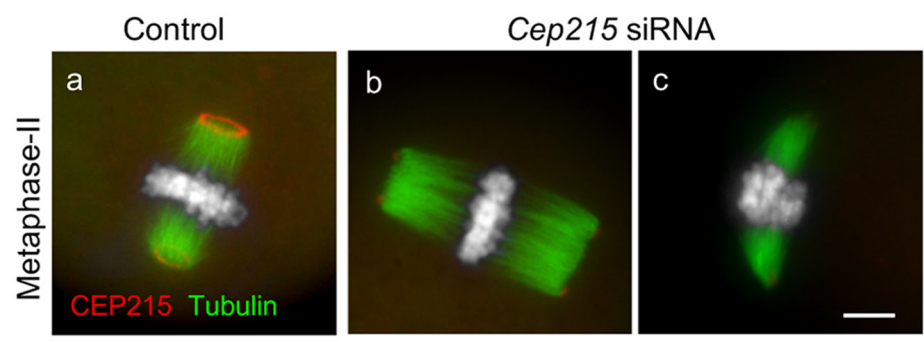

$\mathrm{E}$

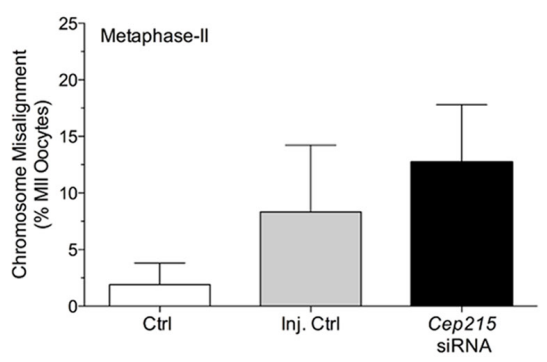

$\mathrm{H}$

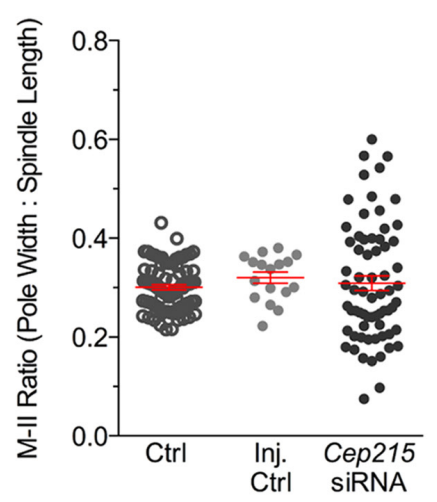

Figure 5 Knockdown of Cep215 transcripts in oocytes disrupts meiotic spindle pole focusing. (A) Fluorescence intensity of CEP215 at the spindle poles in oocytes from the Cep215 siRNA $(n=79)$, non-injected $(n=80)$, and injected control $(n=17)$ groups. (B) Representative images of MII oocytes following in-vitro maturation, double-labeled with anti-CEP215 (red) and anti-acetylated $\alpha$-tubulin antibodies to detect microtubules (green). DAPI labeled DNA is shown in grey. Scale bar of $10 \mu \mathrm{m}$. (C) Percent (mean \pm S.E.M.) of total oocytes that matured to MII after a $17 \mathrm{~h}$ culture. Percentage of MII oocytes that show (D) disrupted meiotic spindle pole focusing and (E) chromosome misalignment errors. Quantitative analysis (mean \pm S.E.M.) of the $(\mathrm{F})$ meiotic spindle length, $(\mathrm{G})$ average pole width, and $(\mathrm{H})$ ratio of the pole width to spindle length between control and Cep215-knockdown MIl-stage oocytes ( $n=4$ replicates). Different letters denote statistical significance at $P<0.05$.

of CEP215 knockdown on the resumption of meiosis. In the non-injected control group, approximately $70 \%$ of oocytes progressed to metaphase-II. Both the injected control and Cep215 siRNA groups showed lower rates $(P<0.05)$ of maturation to MII $(55-58 \%)$, indicating that the microinjection procedure impaired oocyte maturation rates, while Cep215 knockdown had no significant effect.

Disrupted meiotic spindle pole focusing was observed in individual Cep215-knockdown oocytes, both in oocytes that progressed to MII (Fig. 5B) as well as those that remained at $\mathrm{MI}$ (data not shown). After a $17 \mathrm{~h}$ culture, over $90 \%$ of MII-stage oocytes from the control groups contained organized (bipolar, barrel-shaped) meiotic spindles with aligned chromosomes and clear CEP215 labeling at both spindle poles (Fig. 5B and D). In contrast, approximately $40 \%$ of Cep215-knockdown oocytes showed disrupted spindle pole focusing at MII (Fig. 5D). A trend toward increasing chromosome misalignment in the Cep215 siRNA group was also noted, but did not differ significantly from the controls (Fig. 5E). There was no difference in the mean spindle length (distance from pole-to-pole) between the Cep215 siRNA and control groups (Fig. 5F). However, meiotic spindles in Cep215-knockdown oocytes showed high variability in the spindle pole width (Fig. 5G), with both 
broader and narrowly focused spindle poles observed. Thus, the ratio of pole width to spindle length (Fig. $5 \mathrm{H}$ ) was remarkably variable. Oocytes that remained arrested at $\mathrm{MI}$ after the $17 \mathrm{~h}$ culture also showed high variability in the spindle pole width (data not shown). These data indicate that CEP215 plays a role in the regulation of meiotic spindle pole focusing.

\section{Pericentrin and $\gamma$-tubulin localization and fluorescence intensity at aMTOCs are unaltered in Cep215-knockdown oocytes}

Next, we tested whether Cep215-knockdown in oocytes disrupted the localization of other key aMTOC-associated proteins, including pericentrin and $\gamma$-tubulin that are important for microtubule nucleation. Almost all ( 90\%) Cep215-knockdown oocytes showed persistent bright PCNT labeling at aMTOCs (Fig. 6A and B), in a pattern that was similar to the control oocytes. $\gamma$-Tubulin (Fig. 6C and D) was detected along the spindle microtubules near the poles and co-localized with CEP215 at aMTOCs in all control oocytes. Despite markedly low or no CEP215 at aMTOCs, $\gamma$-tubulin labeling persisted at the meiotic spindle poles and microtubules near the poles in Cep215 siRNA-injected oocytes (Fig. 6C and D). There was no difference in either PCNT or $\gamma$-tubulin fluorescence intensity at aMTOCs assessed in individual oocytes, between the
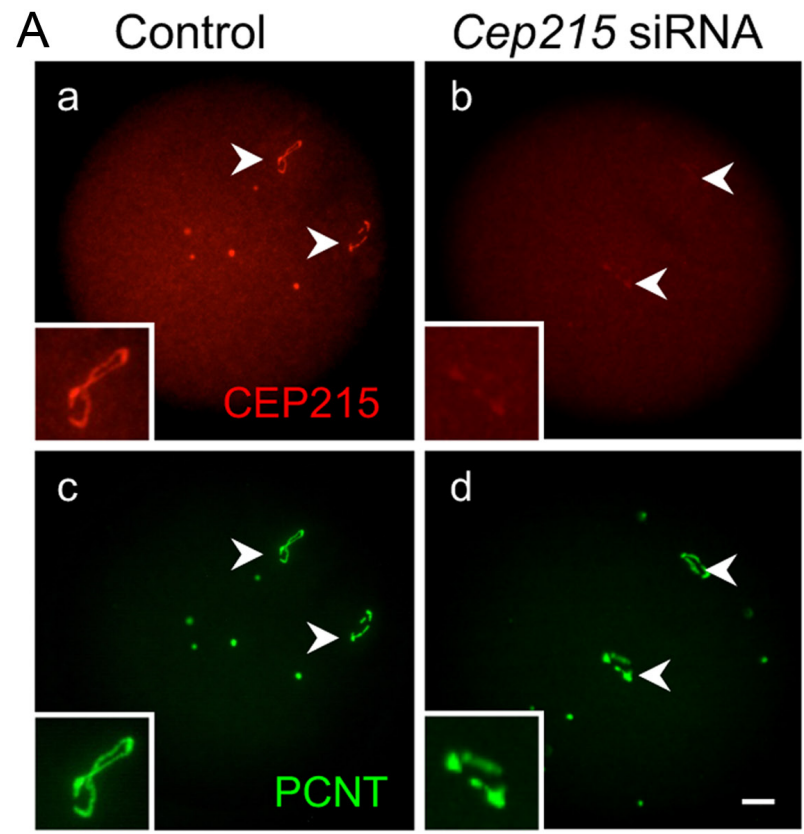

$\mathrm{B}$

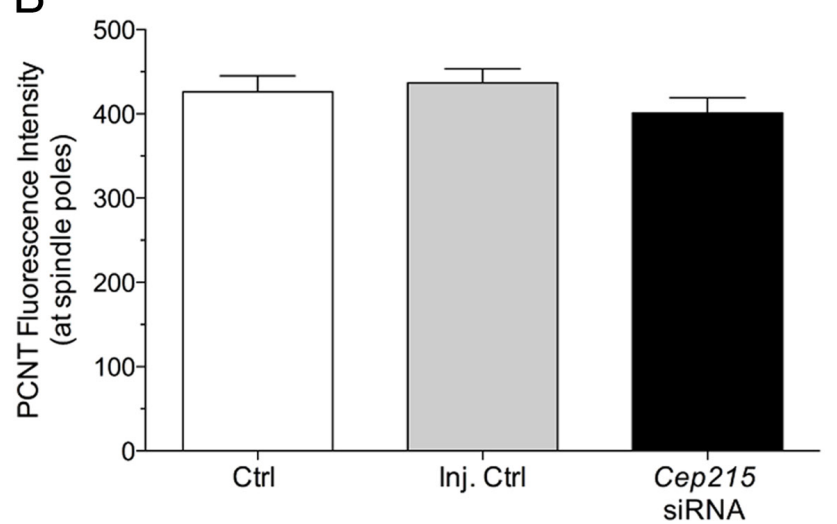

C Control
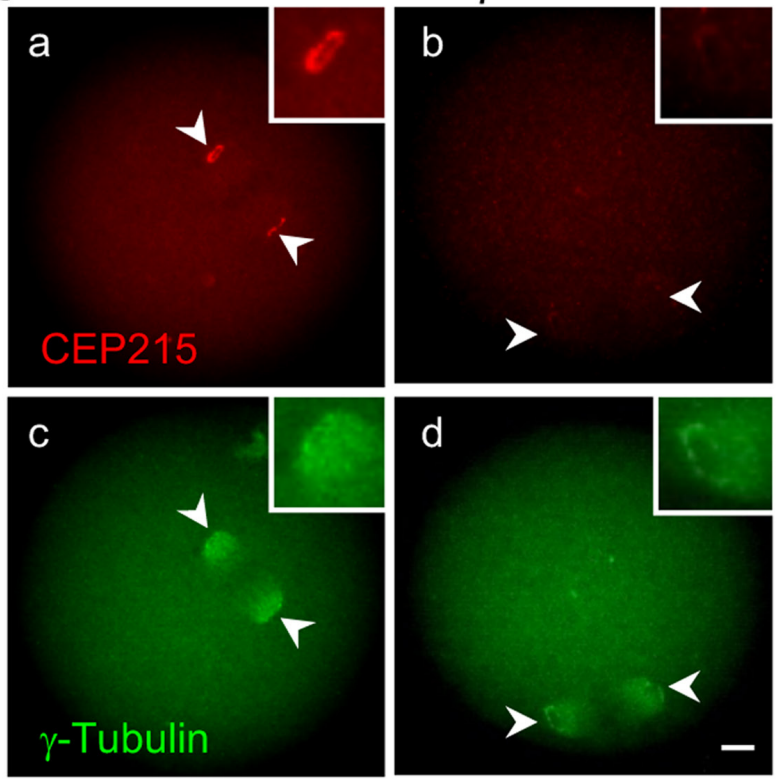

D

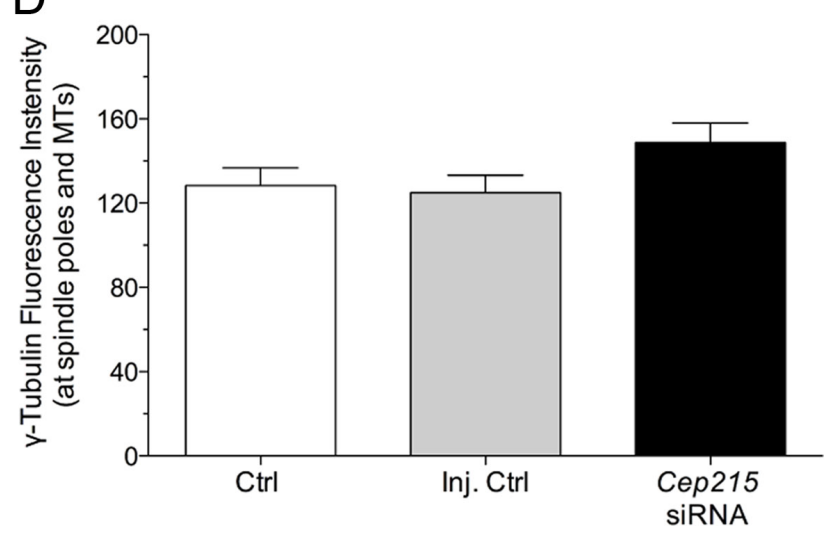

Figure 6 Knockdown of Cep215 transcripts does not disrupt pericentrin or $\gamma$-tubulin localization at oocyte aMTOCs. Representative images of oocytes from both the non-injected $(n=165)$ and injected controls $(n=145)$ compared to the Cep215 siRNA $(n=130)$ group $(n=4$ replicates). (A) Oocytes double-labeled with anti-CEP215 (red, arrows) and anti-PCNT (green, arrows). (B) Fluorescence intensity of PCNT at the spindle poles in oocytes from the Cep215 siRNA and the control groups. (C) Oocytes double-labeled with anti-CEP215 (red, arrows) together with anti- $\gamma$ tubulin (green, arrows). (D) Fluorescence intensity of $\gamma$-tubulin the spindle poles and MTs in oocytes from the Cep215 siRNA and the control groups. Insets show a $2 \times$ magnification of the spindle pole area. Scale bar of $10 \mu \mathrm{m}$. 
control and Cep215 siRNA group (Fig. 6B and D). These data indicate that PCNT and $\gamma$-tubulin localization at the aMTOCs is not critically dependent on CEP215 in mouse oocytes.

\section{Discussion}

In this study, we demonstrate that CEP215 is a functional component of aMTOCs in mouse oocytes and that it localizes to aMTOCs in a pericentrin-dependent manner. Knockdown of Cep215 transcripts in oocytes leads to high variability in meiotic spindle pole focusing, but does not disturb either $\gamma$-tubulin or pericentrin localization at aMTOCs. Thus, indicating a difference in CEP215 function relative to mitotic cells. Moreover, we present novel super-resolution microscopy analysis of oocyte aMTOC organization and uncover an important role for Aurora Kinase A activity in the maintenance of aMTOC organization at assembled meiotic spindles poles.

CEP215 is expressed at aMTOCs in pre-ovulatory oocytes and throughout the progression of meiosis in a pattern similar to other key aMTOC-associated proteins, including pericentrin and the $\gamma$ TuRC-associated protein, NEDD1 (Ma et al. 2010). CEP215 specifically co-localizes with pericentrin at aMTOCs, as reported in somatic cell centrosomes (Fong et al. 2008, Buchman et al. 2010, Choi et al. 2010, Kraemer et al. 2011). Notably, SR-SIM microscopy reveals CEP215 and pericentrin overlap in a unique pore/ring-like organization, with the two proteins detected in a seemingly 'interweaving' pattern. At prophase-I, the aMTOC-associated proteins form a small 'pore-like' structure, whereas at the spindle poles of MII oocytes a partial or complete large ring structure is observed. This ' $\mathrm{O}$ ' or ' $\mathrm{C}$ ' shaped configuration of pericentrin was previously described in MII mouse oocytes, (Carabatsos et al. 2000, Ma et al. 2010). However, this is the first report of the unique pore-like aMTOC organization during prophase-I as well as the high-resolution analysis of pericentrin and CEP215 co-localization. This unique aMTOC configuration is suggestive of a higher order organization of PCM proteins, as described in the centrosomes of somatic cells (Lawo et al. 2012, Mennella et al. 2012, Sonnen et al. 2012). Notably, it remains to be determined how the formation and organization of the 'pore/ring-like' structure of oocyte aMTOCs is regulated, particularly in the absence of centrioles.

CEP215 localization to oocyte aMTOCs is dependent on pericentrin. We previously reported that MII oocytes collected from transgenic mice, with an oocyteconditional Pcnt-knockdown, lack CEP215 at the meiotic spindle poles (Baumann et al. 2017). In the current study, we expanded on this analysis to test potential CEP215/PCNT interaction at the GV-stage and compared total CEP215 between control and transgenic oocytes. Notably, we show that CEP215 is absent in aMTOCs in Pcnt-depleted oocytes at both GV and
MII-stages. Nevertheless, total CEP215 levels are unchanged in Pcnt-depleted MII oocytes, demonstrating that the loss of PCNT specifically disrupts the localization of CEP215 to aMTOCs and not the overall protein levels. These findings are comparable to observations in somatic cells, where CEP215 targeting to centrosomes relies on PCNT (Graser et al. 2007, Fong et al. 2008) irrespective of the phosphorylation status of PCNT (Lee \& Rhee 2011). Our current experiments also demonstrate that CEP215 distribution at aMTOCs is regulated by microtubules. Blocking microtubule polymerization in oocytes (with a brief exposure to nocodazole) during the resumption of meiosis leads to a lack of microtubules around the chromosomes and only one or two CEP215labelled aMTOCs, contrasting with multiple aMTOCs detected in untreated oocytes. Only after nocodazole removal and MT formation did the total number of CEP215 foci increase significantly in the treated oocytes and gradually reorganize. This is consistent with earlier studies of PCNT live cell imaging, which showed that aMTOC fragmentation and organization upon meiotic resumption is microtubule dependent (Schuh \& Ellenberg 2007, Clift \& Schuh 2015). In sum, our data support that CEP215 is a conserved protein component of aMTOCs in mammalian oocytes at prophase-I and during meiotic maturation.

We also tested whether Aurora Kinase A activity regulates CEP215 distribution at assembled meiotic spindle pole aMTOCs in both MI and MII oocytes. Phosphorylated Aurora A localizes to aMTOCs and contributes to meiotic spindle assembly during meiosis I in mouse oocytes (Solc et al. 2012, Bury et al. 2017). Studies in Drosophila also show that Aurora A participates in centrosome maturation during mitotic division, in part, by regulating CEP215-mediated targeting of $\gamma$-tubulin to the centrosome (Terada et al. 2003). In mouse oocytes, depletion or inhibition of Aurora A reportedly delays initial MT nucleation and establishment of a bipolar spindle at MI as well as progression to MII (Ding et al. 2011, Solc et al. 2012, Bury et al. 2017). Hence, we aimed to understand whether Aurora A activity affects CEP215 localization specifically in assembled $\mathrm{MI}$ and MII spindles. Pharmacological inhibition of Aurora A did not reduce overall CEP215 fluorescence intensity levels at aMTOC in either MI or MII oocytes, as previously reported in Drosophila centrosomes (Terada et al. 2003), indicating that the kinase is unlikely to play a critical role in the maintenance of CEP215 at oocyte aMTOCs at assembled spindle poles. Intriguingly, however, we found that Aurora A inhibition leads to pronounced CEP215 and PCNT clustering into small foci at each spindle pole as well as a striking loss of the aMTOC ring-like structural organization in both $\mathrm{MI}$ and MIl-stage oocytes. This occurs in conjunction with tight focusing of minus-end microtubules into narrow spindle poles and shorter spindle lengths, suggestive of disrupted microtubule polymerization and/or 
stabilization. The observed increase in chromosome errors may also be potentially linked to microtubule instability since AURKA promotes microtubule nucleation (Sardon et al. 2008). Smaller meiotic spindles have also been reported in oocytes that specifically express a catalytically inactive AURKA (Solc et al. 2012). Interestingly, in somatic cells AURKA phosphorylates TPX2, which is essential for normal microtubule flux to maintain spindle length at metaphase (Fu et al. 2015). Studies in mouse oocytes likewise show that AURKA contributes to rapid microtubule growth at the initial stages of meiotic spindle formation during meiosis I (Bury et al. 2017).

Disruption of aMTOCs can also affect spindle structure. We previously established that loss of aMTOCs in PCNT-depleted oocytes leads to the formation of shorter spindles (Baumann et al. 2017). Moreover, a recent study demonstrated that AURKA and its downstream target, TACC3, are essential for assembly of the newly identified 'liquid-like meiotic spindle domain' (LISD) in mammalian oocytes. The LISD reportedly serves as a reservoir for microtubule regulatory factors in proximity to the spindle poles and microtubules, and it promotes stable meiotic spindle assembly (So et al. 2019). Notably, LISD disruption by AURKA inhibition or TACC3 depletion led to significantly altered MT dynamics, reduced spindle volumes, and tightly focused aMTOCs at the spindle poles. This is similar to our observations and points to a potential mechanism by which AURKA activity and/or the LISD may regulate meiotic spindle and aMTOC organization. Taken together, our current data reveal a critical role for AURKA activity in regulating microtubule dynamics, spindle pole focusing, and the maintenance of oocyte aMTOC organization at assembled meiotic spindles. Further studies are needed to gain a better understanding of how the loss of aMTOC ring-structure organization may be directly or indirectly linked to hyper focusing of the spindle poles as well as to address the potential mechanisms of action.

CEP215 function was assessed by siRNA-mediated transcript knockdown that effectively reduced CEP215 fluorescence intensity at oocyte aMTOCs. Knockdown of Cep215 transcripts did not block the resumption of meiosis, oocyte progression to MII, nor meiotic spindle length. Nevertheless, CEP215-deficient oocytes exhibit defects in the regulation of spindle pole focusing. Studies in human cell lines report that CEP215 may function together with ASPM (abnormal spindle-like microcephaly-associated protein) in the regulation of spindle pole focusing (Tungadi et al. 2017). ASPM also plays a role in spindle pole assembly in C. elegans oocytes (Connolly et al. 2014) and is expressed in mouse oocytes where its knockdown disrupts meiotic spindle organization (Xu et al. 2012). Whether there is an interaction between CEP215 and ASPM in mouse oocytes remains to be determined. Notably, disruption of spindle pole focusing in Cep215-knockdown oocytes did not correlate with changes in either pericentrin or $\gamma$-tubulin localization and fluorescence intensity at aMTOCs. Pericentrin was detected at aMTOCs of knockdown oocytes, indicating that CEP215 does not play a major role in the localization of this core scaffolding protein to oocyte aMTOCs. In somatic cells, reports vary as to whether CEP215 plays a major role in regulating pericentrin targeting to centrosomes (Fong et al. 2008, Kim \& Rhee 2014). Surprisingly, $\gamma$-tubulin distribution at aMTOCs is also comparable between control and Cep215-knockdown oocytes. This differs from somatic cells, where CEP215 has been shown to interact with $\gamma$ TuRCs at the centrosomes throughout the cell cycle (Fong et al. 2008, Choi et al. 2010), and its depletion reduces $\gamma$-tubulin at the centrosome as well as microtubule nucleation (Choi et al. 2010, Muroyama et al. 2016). Hence, while pericentrin is essential for $\gamma$-tubulin accumulation at oocyte aMTOCs (Ma \& Viveiros 2014, Baumann et al. 2017), our current data suggests that CEP215 alone is not critical for this process, and further studies are warranted to address this potential difference.

Taken together the current experiments support that CEP215 is a functional component of aMTOCs in mouse oocytes and plays role in regulating meiotic spindle pole focusing. Notably, we show differences in oocyte CEP215 function relative to mitosis, and also reveal an important role for Aurora Kinase A activity in the maintenance of oocyte aMTOC organization at assembled meiotic spindles poles.

\section{Supplementary materials}

This is linked to the online version of the paper at https://doi. org/10.1530/REP-19-0263.

\section{Declaration of interest}

The authors declare that there is no conflict of interest that could be perceived as prejudicing the impartiality of the research reported.

\section{Funding}

This work was supported by a grant (HD092857) from the Eunice Kennedy Shriver National Institute of Child Health and Human Development (M MV) and a Graduate Fellowship from the Department of Physiology and Pharmacology, University of Georgia (X W). R D L F and C B supported by NIH (HD093383) and NSF Center for Cell Manufacturing (CMaT).

\section{Author contribution statement}

X W performed experiments, analyzed data, and co-wrote the manuscript. C B assisted with experiments. R D L F contributed to experiment design and manuscript preparation 
as well as provided reagents. M M V conceived and designed experiments, analyzed data, wrote the manuscript, and supervised the research.

\section{Acknowledgements}

The authors thank Dr M K Kandasamy for his assistance with high resolution SR-SIM microscopy analysis at the Biomedical Microscopy Core facility, University of Georgia. Moreover, they thank lab members, Luhan Yang and Tiffany Yue for their assistance and manuscript review.

\section{References}

Baumann C \& Viveiros MM 2015 Meiotic spindle assessment in mouse oocytes by siRNA-mediated silencing. Journal of Visualized Experiments 104 e53586. (https://doi.org/10.3791/53586)

Baumann C, Wang X, Yang L \& Viveiros MM 2017 Error-prone meiotic division and subfertility in mice with oocyte-conditional knockdown of pericentrin. Journal of Cell Science 130 1251-1262. (https://doi. org/10.1242/jcs.196188)

Bond J, Roberts E, Springell K, Lizarraga SB, Scott S, Higgins J, Hampshire DJ, Morrison EE, Leal GF, Silva EO et al. 2005 A centrosomal mechanism involving CDK5RAP2 and CENPJ controls brain size. Nature Genetics 37 353-355. (https://doi.org/10.1038/ng1539)

Buchman JJ, Tseng HC, Zhou Y, Frank CL, Xie Z \& Tsai LH 2010 Cdk5rap2 interacts with pericentrin to maintain the neural progenitor pool in the developing neocortex. Neuron 66 386-402. (https://doi.org/10.1016/j. neuron.2010.03.036)

Bury L, Coelho PA, Simeone A, Ferries S, Eyers CE, Eyers PA, ZernickaGoetz M \& Glover DM 2017 Plk4 and Aurora A cooperate in the initiation of acentriolar spindle assembly in mammalian oocytes. Journal of Cell Biology 216 3571-3590. (https://doi.org/10.1083/jcb.201606077)

Carabatsos MJ, Combelles CMH, Messinger SM \& Albertini DF 2000 Sorting and reorganization of centrosomes during oocyte maturation in the mouse. Microscopy Research and Technique 49 435-444. (https://doi.org/10.1002/(SICI)1097-0029(20000601)49:5<435::AIDJEMT5>3.0.CO;2-H)

Chen CT, Hehnly $H$, Yu Q, Farkas D, Zheng G, Redick SD, Hung HF, Samtani R, Jurczyk A, Akbarian S et al. 2014 A unique set of centrosome proteins requires pericentrin for spindle-pole localization and spindle orientation. Current Biology 24 2327-2334. (https://doi.org/10.1016/j. cub.2014.08.029)

Chmátal L, Yang K, Schultz RM \& Lampson MA 2015 Spatial regulation of kinetochore microtubule attachments by destabilization at spindle poles in meiosis I. Current Biology 25 1835-1841. (https://doi.org/10.1016/j. cub.2015.05.013)

Choi YK, Liu P, Sze SK, Dai C \& Qi RZ 2010 CDK5RAP2 stimulates microtubule nucleation by the $\gamma$-tubulin ring complex. Journal of Cell Biology 191 1089-1095. (https://doi.org/10.1083/jcb.201007030)

Clift D \& Schuh M 2015 A three-step MTOC fragmentation mechanism facilitates bipolar spindle assembly in mouse oocytes. Nature Communications 6 7217. (https://doi.org/10.1038/ncomms8217)

Combelles CMH \& Albertini DF 2001 Microtubule patterning during meiotic maturation in mouse oocytes is determined by cell cyclespecific sorting and redistribution of g-tubulin. Developmental Biology 239 281-294. (https://doi.org/10.1006/dbio.2001.0444)

Connolly AA, Osterberg V, Christensen S, Price M, Lu C, Chicas-Cruz K, Lockery S, Mains PE \& Bowerman B 2014 Caenorhabditis elegans oocyte meiotic spindle pole assembly requires microtubule severing and the calponin homology domain protein ASPM-1. Molecular Biology of the Cell 25 1298-1311. (https://doi.org/10.1091/mbc.E13-11-0687)

Delaval B \& Doxsey SJ 2010 Pericentrin in cellular function and disease. Journal of Cell Biology 188 181-190. (https://doi.org/10.1083/ jcb.200908114)

Ding J, Swain JE \& Smith GD 2011 Aurora kinase-A regulates microtubule organizing center (MTOC) localization, chromosome dynamics, and histone-H3 phosphorylation in mouse oocytes. Molecular Reproduction and Development 78 80-90. (https://doi.org/10.1002/mrd.21272)
Doxsey S, McCollum D \& Theurkauf W 2005 Centrosomes in cellular regulation. Annual Review of Cell and Developmental Biology 21 411-434. (https://doi.org/10.1146/annurev.cellbio.21.122303.120418)

Fong KW, Choi YK, Rattner JB \& Qi RZ 2008 CDK5RAP2 is a pericentriolar protein that functions in centrosomal attachment of the $\gamma$-tubulin ring complex. Molecular Biology of the Cell 19 115-125. (https://doi. org/10.1091/mbc.e07-04-0371)

Fu J, Bian M, Xin G, Deng Z, Luo J, Guo X, Chen H, Wang Y, Jiang Q \& Zhang C 2015 TPX2 phosphorylation maintains metaphase spindle length by regulating microtubule flux. Journal of Cell Biology $\mathbf{2 1 0}$ 373-383. (https://doi.org/10.1083/jcb.201412109)

Graser S, Stierhof YD \& Nigg EA 2007 Cep68 and Cep215 (Cdk5rap2) are required for centrosome cohesion. Journal of Cell Science $\mathbf{1 2 0}$ 4321-4331. (https://doi.org/10.1242/jcs.020248)

Hassold TJ \& Hunt PA 2001 To err (meiotically) is human: the genesis of human aneuploidy. Nature Reviews: Genetics 2 280-291. (https://doi. org/10.1038/35066065)

Kim S \& Rhee K 2014 Importance of the CEP215-pericentrin interaction for centrosome maturation during mitosis. PLOS ONE 9 e87016. (https://doi. org/10.1371/journal.pone.0087016)

Kraemer N, Issa L, Hauck SCR, Mani S, Ninnemann O \& Kaindl AM 2011 What's the hype about CDK5RAP2? Cellular and Molecular Life Sciences 68 1719-1736. (https://doi.org/10.1007/s00018-011-0635-4)

Lawo S, Hasegan M, Gupta GD \& Pelletier L 2012 Subdiffraction imaging of centrosomes reveals higher-order organizational features of pericentriolar material. Nature Cell Biology 14 1148-1158. (https://doi. org/10.1038/ncb2591)

Lee K \& Rhee K 2011 PLK1 phosphorylation of pericentrin initiates centrosome maturation at the onset of mitosis. Journal of Cell Biology 195 1093-1101. (https://doi.org/10.1083/jcb.201106093)

Lizarraga SB, Margossian SP, Harris MH, Campagna DR, Han AP, Blevins S, Mudbhary R, Barker JE, Walsh CA \& Fleming MD 2010 Cdk5rap2 regulates centrosome function and chromosome segregation in neuronal progenitors. Development 137 1907-1917. (https://doi.org/10.1242/ dev.040410)

Ma W \& Viveiros MM 2014 Depletion of pericentrin in mouse oocytes disrupts microtubule organizing center function and meiotic spindle organization. Molecular Reproduction and Development $\mathbf{8 1}$ 1019-1029. (https://doi.org/10.1002/mrd.22422)

Ma W, Baumann C \& Viveiros MM 2010 NEDD1 is crucial for meiotic spindle stability and accurate chromosome segregation in mammalian oocytes. Developmental Biology 339 439-450. (https://doi.org/10.1016/j. ydbio.2010.01.009)

Manandhar G, Schatten H \& Sutovsky P 2005 Centrosome reduction during gametogenesis and its significance. Biology of Reproduction $\mathbf{7 2}$ 2-13. (https://doi.org/10.1095/biolreprod.104.031245)

Maro B, Howlett SK \& Webb M 1985 Non-spindle microtubule organizing centers in metaphase II-arrested mouse oocytes. Journal of Cell Biology 101 1665-1672. (https://doi.org/10.1083/jcb.101.5.1665)

Mennella V, Keszthelyi B, McDonald KL, Chhun B, Kan F, Rogers GC, Huang B \& Agard DA 2012 Subdiffraction-resolution fluorescence microscopy reveals a domain of the centrosome critical for pericentriolar material organization. Nature Cell Biology 14 1159-1168. (https://doi. org/10.1038/ncb2597)

Muroyama A, Seldin L \& Lechler T 2016 Divergent regulation of functionally distinct $\gamma$-tubulin complexes during differentiation. Journal of Cell Biology 213 679-692. (https://doi.org/10.1083/jcb.201601099)

Nagaoka SI, Hassold TJ \& Hunt PA 2012 Human aneuploidy: mechanisms and new insights into an age-old problem. Nature Reviews: Genetics 13 493-504. (https://doi.org/10.1038/nrg3245)

Russell ES, McFarland EC \& Peters H 1985 Gametic and pleiotropic defects in mouse fetuses with Hertwig's macrocytic anemia. Developmental Biology 110 331-337. (https://doi.org/10.1016/0012-1606(85)90092-2)

Sardon T, Peset I, Petrova B \& Vernos I 2008 Dissecting the role of Aurora A during spindle assembly. EMBO Journal 27 2567-2579. (https://doi. org/10.1038/emboj.2008.173)

Sathananthan AH, Selvaraj K \& Trounson A 2000 Fine structure of human oogonia in the foetal ovary. Molecular and Cellular Endocrinology 161 3-8. (https://doi.org/10.1016/s0303-7207(99)00216-6)

Schuh M \& Ellenberg J 2007 Self-organization of MTOCs replaces centrosome function during acentrosomal spindle assembly in live mouse oocytes. Cell 130 484-498. (https://doi.org/10.1016/j.cell.2007.06.025) 
Sloane DA, Trikic MZ, Chu MLH, Lamers MB, Mason CS, Mueller I, Savory WJ, Williams DH \& Eyers PA 2010 Drug-resistant Aurora A mutants for cellular target validation of the small molecule kinase inhibitors MLN8054 and MLN8237. ACS Chemical Biology 5 563-576. (https://doi.org/10.1021/cb100053q)

So C, Seres KB, Steyer AM, Monnich E, Clift D, Pejkovska A, Mobius W \& Schuh M 2019 A liquid-like spindle domain promotes acentrosomal spindle assembly in mammalian oocytes. Science 364 eaat9557. (https:// doi.org/10.1126/science.aat9557)

Solc P, Baran V, Mayer A, Bohmova T, Panenkova-Havlova G, Saskova A, Schultz RM \& Motlik J 2012 Aurora kinase A drives MTOC biogenesis but does not trigger resumption of meiosis in mouse oocytes matured in vivo. Biology of Reproduction 87 85-85. (https://doi.org/10.1095/ biolreprod.112.101014)

Sonnen KF, Schermelleh L, Leonhardt H \& Nigg EA 2012 3D-structured illumination microscopy provides novel insight into architecture of human centrosomes. Biology Open 1 965-976. (https://doi.org/10.1242/ bio.20122337)

Szollosi D, Calarco P \& Donahue RP 1972 Absence of centrioles in the first and second meiotic spindles of mouse oocytes. Journal of Cell Science $11521-541$.

Terada Y, Uetake Y \& Kuriyama R 2003 Interaction of Aurora-A and centrosomin at the microtubule-nucleating site in Drosophila and mammalian cells. Journal of Cell Biology 162 757-763. (https://doi. org/10.1083/jcb.200305048)
Tungadi EA, Ito A, Kiyomitsu T \& Goshima G 2017 Human microcephaly ASPM protein is a spindle pole-focusing factor that functions redundantly with CDK5RAP2. Journal of Cell Science 130 3676-3684. (https://doi. org/10.1242/jcs.203703)

Xu XL, Ma W, Zhu YB, Wang C, Wang BY, An N, An L, Liu Y, Wu ZH \& Tian JH 2012 The microtubule-associated protein ASPM regulates spindle assembly and meiotic progression in mouse oocytes. PLOS ONE 7 e49303. (https://doi.org/10.1371/journal.pone.0049303)

Zaqout S, Bessa P, Krämer N, Stoltenburg-Didinger G \& Kaindl AM 2017 CDK5RAP2 is required to maintain the germ cell pool during embryonic development. Stem Cell Reports 8 198-204. (https://doi.org/10.1016/j. stemcr.2017.01.002)

Zimmerman WC, Sillibourne J, Rosa J \& Doxsey SJ 2004 Mitosis-specific anchoring of gamma tubulin complexes by pericentrin controls spindle organization and mitotic entry. Molecular Biology of the Cell 15 3642-3657. (https://doi.org/10.1091/mbc.e03-11-0796)

Received 14 June 2019

First decision 10 July 2019

Revised manuscript received 20 December 2019

Accepted 2 January 2020 